\title{
Comparison of Sitting Balance and Coordination in Children with Spastic Cerebral Palsy Using the Korean Version of Trunk Impairment Scale (K-TIS)
}

\author{
Hye-Rim Jung, OT, PhD • Young-Eun Choi, PT, $\mathrm{PhD}^{1 \dagger}$
}

Department of Occupational Therapy, College of Health Medicine, Kaya University

${ }^{1}$ Department of Physical Therapy, College of Health Medicine, Kaya University

Received: September 28, 2019 / Revised: September 1, 2019 / Accepted: October 28, 2019

(C) 2019 J Korean Soc Phys Med

\section{| Abstract |}

PURPOSE: The purpose of this study was to compare sitting balance and coordination spastic cerebral palsy in children using the Korean version of Trunk Impairment Scale (K-TIS) as well as to provide basic data about effective postural control treatment for clinicians handling these two types.

METHODS: The K-TIS was measured in 29 children diagnosed with diplegic and quadriplegic cerebral palsy (18 with diplegia and 11 with quadriplegia). The average and standard scores of the children's K-TIS subscales and items of the two groups were measured. The two groups' subscales and items were analyzed by using the Mann-Whitney U test. RESULTS: Static sitting balance, dynamic sitting balance, coordination, and total score for children with diplegia were statistically high $(\mathrm{p}<.05)$. For all items under static sitting balance, the score for children with diplegia was higher. The

$\uparrow$ Corresponding Author : Young-Eun Choi

choiye00@naver.com, https://orcid.org/0000-0002-2487-5122

This is an Open Access article distributed under the terms of the Creative Commons Attribution Non-Commercial License (http://creativecommons.org/licenses/by-nc/3.0) which permits unrestricted non-commercial use, distribution, and reproduction in any medium, provided the original work is properly cited. first differences in the repeated items of dynamic sitting balance and coordination area that rotates between the upper and lower body were presented.

CONCLUSION: The difference in balance and coordination in sitting positions is exhibited in children with diplegia and quadriplegia. For children with spastic quadriplegia, treatments should focus on static sitting balance and coordination, together with a focus on dynamic sitting balance and coordination.

Key Words: Cerebral palsy, Diplegia, Quadriplegia, Sitting balance, Postural control

\section{Introduction}

Cerebral palsy, a permanent non-progressive disorder affecting the brain of a fetus or child, impairs the development of movement and posture. The motor disorders of cerebral palsy are often accompanied by issues in sensation, perception, cognition, communication, behavior, epilepsy, and secondary musculoskeletal problems [1]. These occurrences lead to abnormal posture, loss of selective motor control, and poor trunk control and balance, which contributes to poor postural control and results in limited activities in daily life [2].

Balance and postural control are the basic components 
of motor skills, which are predictable, spontaneous, and necessary to maintain the equilibrium of the body in a specific environment $[3,4]$. Children with cerebral palsy have problems with muscle weakness, muscle tone, and sensory information input, which lead to difficulty in maintaining trunk stability, postural control [5], postural alignment, antigravity muscles, equilibrium, and midline [6]. Problems with interactions between the sensory system, central nervous system, and musculoskeletal system make it difficult to maintain stable postural control. In addition, errors with the sequence of muscle recruitment lead to a reduction in coordination $[7,8]$. Spastic cerebral palsy is the most common type of cerebral palsy, which manifests through abnormal postural tone, motor function impairments $[9,10]$, and difficulty in maintaining static trunk control, all of which can affect one's sitting or standing posture [11].

Diplegia and quadriplegia account for a large percentage of spastic cerebral palsy. The commonality between the two conditions is that they both include impairments to lower extremities. Mild impairments in white matter are limited to fibers, which are closest to the brain ventricle that sends signals to the lower extremities. Severe impairments or impairments tilted sideways in white matter affect upper extremity fibers [12], and this is categorized as diplegia or quadriplegia. As quadriplegia is associated with an extensive range of impairments and problems in both upper extremities, it may affect gray matter injuries, dyskinesia, epilepsy, and cognitive functions [13].

Proper classification of cerebral palsy is important because there is a close correlation between the anatomical distribution of impairments and appropriate clinical treatments based on an understanding of the interactions between impairment and development. In a prior study that compared trunk control among children with cerebral palsy based on the Trunk Control Measurement Scale (TCMS) [11], hemiplegic children scored the highest, followed by diplegic and quadriplegic children. Their TCMS noticeably decreased in accordance with an increasing level in the Gross Motor Function Classification System (GMFCS). Accurately understanding movement characteristics, posture, balance, and coordination based on differences in upper and lower extremity functions between diplegia and quadriplegia is essential in order to identify optimal treatments for children with cerebral palsy in terms of exercises and training activities. In Korea and abroad, there have been limited studies that compare diplegic and quadriplegic children by measuring their trunk control and balance with an objective scale. The difference in trunk balance between quadriplegia and diplegia is predictable and has already been shown through clinical observation and some measurement tools. First, this paper aimed to identify and describe the differences in trunk function that are easily observed in a clinical setting using reliable evaluation tools. Second, it aimed to determine whether or not the Korean version of TIS (K-TIS) is able to discriminate trunk function between the two groups and represent differences in items. In overseas studies, the reliability and validity of K-TIS were investigated in the context of cerebral palsy. In order to determine the validity of GMFCS, some studies have compared the functions of clinically predicted cerebral palsy groups. Therefore, this study describes the differences between the two groups based on K-TIS items and provides preliminary data for the use and treatment of K-TIS by clinicians.

\section{Methods}

\section{Study subjects}

The present study was conducted on 29 children with static diplegia and quadriplegia (18 with diplegia and 11 with quadriplegia) from June 2019 to July 2019, at Hospital B, Gyeonggi-do, Korea. The children's representatives (guardians) were fully informed of the purpose of the study and any risks that may arise during their participation, after which they were asked to sign their written consent. 
Inclusion criteria included (1) children aged 6 to 12 years diagnosed with static diplegia and quadriplegia by a physician, (2) children capable of following the therapist's instructions, and (3) children with no history of any operation or surgery in the last 6 months. Exclusion criteria included (1) children with congenital musculoskeletal disorders, progressive central nervous system diseases, or a history of orthopedic surgery and (2) children with genetic diseases and severe diseases other than cerebral palsy.

The K-TIS was administered by a physical therapist with 7 years of experience through a one-on-one session with the participant in a familiar and quiet treatment room in order to avoid any errors in measurement. During measurements, the children were asked to wear the shoes or orthotics they usually used, and the height of the mat was adjusted to make sure that their feet were supported on the surface and that their pelvis and knee joints were maintained at a $90^{\circ}$ angle.

\section{Measurement}

The TIS is a scale that has been developed and tested for its reliability to evaluate balance and trunk coordination in stroke patients' sitting posture [14]. The TIS consists of three subscales (static sitting balance, dynamic sitting balance, and coordination) and 17 items, with scores ranging from 0 to 23 . The static sitting balance test consists of three items: maintaining a sitting posture with feet supported, maintaining a sitting posture with legs crossed by the therapist, and maintaining a sitting posture with legs crossed by the patient. It assigns 0 to 2 points or 0 to 3 points to each item, which results in a score of 0 to 7 . On the other hand, the Dynamic sitting balance test consists of 10 items to evaluate bending of the trunk to the side and lifting of either side of the pelvis. It assigns 0 to 1 point to each item, which results in a score of 0 to 10 in total. Lastly, the coordination test consists of four items to evaluate rotation of the upper or lower part of his or her trunk six times and assigns 0 to 1 point or
0 to 2 points to each item, which results in a score of 0 to 6 in total. The present study used the Korean Version of the Trunk Impairment Scale (K-TIS) modified by Ko and You [15]. The scale's inter-rater reliability was ICC3, $1=.920-.983$ ( $\mathrm{r}=.924 .984)$, and its test-retest reliability was ICC3,1=.805-.901 ( $\mathrm{r}=.806-.903)$.

\section{Statistical analyses}

Frequency analysis and descriptive statistics were used for analysis of the participating children's demographic characteristics and affected areas. The children's K-TIS average and standard scores were analyzed by descriptive statistics, whereas the two groups' subscales and items were analyzed by using the Mann-Whitney U test. An SPSS 21 version was used as a statistical program, and $p<.05$ was considered to indicate statistical significance.

\section{Results}

\section{General characteristics of the participants}

There was a total of 29 participants, and Table 1 shows the general characteristics and differences between the two groups.

\section{Differences in K-TIS subscales between children} with diplegia and quadriplegia

Table 2 provides the score in TIS subscales between children with diplegia and quadriplegia.

\section{Differences in K-TIS items between children} with diplegia and quadriplegia

Tables 3, 4, and 5 show the scores of items under the K-TIS subscales between children with diplegia and quadriplegia.

\section{Discussion}

The TIS used in the present study showed internal co 
Table 1. General Characteristics of the Participants

\begin{tabular}{cccc}
\hline \multirow{2}{*}{ Variables } & & \multicolumn{2}{c}{ Type of Cerebral Palsy } \\
\cline { 3 - 4 } & & Diplegia & Quadriplegia \\
\hline Gender, n (\%) & Male & $9(50)$ & $2(18.2)$ \\
& Female & $9(50)$ & $9(81.8)$ \\
\hline Age (year), mean \pm SD & 1 & $7.83 \pm 1.68$ & $9.18 \pm 1.66$ \\
\hline \multirow{2}{*}{ GMFCS, n (\%) } & 2 & $5(27.8)$ & $0(.0)$ \\
& 3 & $6(33.3)$ & $0(.0)$ \\
& 4 & $7(38.9)$ & $6(.0)$ \\
& 5 & $0(.0)$ & $5(44.5)$ \\
\hline Total, $\mathrm{n}(\%)$ & & $18(.0)$ & $11(33.3)$ \\
\hline
\end{tabular}

n: number, mean \pm SD: Mean \pm standard deviation, GMFCS: Gross motor function classification system

Table 2. Difference Between Two Groups in the K-TIS

\begin{tabular}{cccccc}
\hline K-TIS & Diplegia & Quadriplegia & difference & $\mathrm{z}$ & $\mathrm{p}$ \\
\hline Static Sitting Balance & $6.33 \pm .97$ & $1.82 \pm 2.22$ & 4.51 & -4.239 & $<.001^{* *}$ \\
Dynamic Sitting Balance & $5.83 \pm .85$ & $1.82 \pm 2.31$ & 4.01 & -3.268 & $.001^{* *}$ \\
Coordination & $3.11 \pm .96$ & $1.27 \pm 1.67$ & 1.84 & -2.463 & $<.001^{* *}$ \\
\hline Total & $15.28 \pm 5.26$ & $4.91 \pm 6.14$ & 10.37 & -3.393 & $<.001^{* *}$ \\
\hline
\end{tabular}

Values are mean \pm standard deviation, K-TIS: Korean version of trunk impairment scale ${ }^{*} \mathrm{p}<.05,{ }^{* *} \mathrm{p}<.01$

Table 3. Difference Between Two Groups in the Items for Static Sitting Balance

\begin{tabular}{ccccccc}
\hline Item & Diplegia & Quadriplegia & Difference & $\mathrm{z}$ & $\mathrm{p}$ \\
\hline 1 & $2.00 \pm .00$ & $.91 \pm 1.04$ & 1.09 & -3.457 & $.001^{* *}$ \\
2 & $1.78 \pm .64$ & $.36 \pm .80$ & 1.42 & -3.741 & $<.001^{* *}$ \\
3 & $2.39 \pm .60$ & $.55 \pm .82$ & 1.84 & -4.098 & $<.001^{* *}$ \\
\hline
\end{tabular}

Values are mean \pm standard deviation ${ }^{*} \mathrm{p}<.05,{ }^{* *} \mathrm{p}<.01$

nsistency reliability (2.55-3.82) and inter-rater reliability (4.07-8.23) in children aged 5 to 19 years with cerebral palsy in conjunction with a high level of concurrent validity $(\mathrm{r}=.80-.87)$. In a correlation comparison with the Gross Motor Function Measure (GMFM), the scale was deemed suitable for evaluation of balance and coordination in children in the present study's age range [16].
In general, diplegic children have a higher level of gross motor skills than quadriplegic children. In addition, the GMFCS level of diplegic children was higher than that of quadriplegic children $(\mathrm{p}=0.00)$. This is consistent with the results of Beckung et al. [17], which reported $72 \%$ of diplegic participants had a GMFCS level of I to III, whereas $100 \%$ of the quadriplegia participants were at level 
Table 4. Difference Between Two Groups in Items for Dynamic Sitting Balance

\begin{tabular}{cccccc}
\hline Item & Diplegia & Quadriplegia & Difference & \multicolumn{2}{c}{$\mathrm{z}$} \\
\hline 1 & $1.00 \pm .00$ & $.45 \pm .52$ & .55 & -3.457 & $.001^{* *}$ \\
2 & $.72 \pm .46$ & $.27 \pm .46$ & .45 & -2.321 & $.020^{*}$ \\
3 & $.39 \pm .50$ & $.00 \pm .00$ & .39 & -2.333 & $.020^{*}$ \\
4 & $.94 \pm .23$ & $.45 \pm .52$ & .49 & -2.939 & $.003^{* *}$ \\
5 & $.67 \pm .48$ & $.27 \pm .46$ & .40 & -2.024 & $.043^{*}$ \\
6 & $.39 \pm .50$ & $.00 \pm .00$ & .39 & -2.333 & $.020^{*}$ \\
7 & $.56 \pm .51$ & $.09 \pm .30$ & .47 & -2.459 & $.014^{*}$ \\
8 & $.22 \pm .42$ & $.00 \pm .00$ & .22 & -1.655 & .098 \\
9 & $.72 \pm .46$ & $.27 \pm .46$ & .45 & -2.321 & $.020^{*}$ \\
10 & $.22 \pm .42$ & $.00 \pm .00$ & .22 & -1.655 & .098 \\
\hline
\end{tabular}

Values are mean \pm standard deviation

${ }^{*} \mathrm{p}<.05, \stackrel{*}{*} \mathrm{p}<.01$

Table 5. Difference Between Two Groups in the Items of Coordination

\begin{tabular}{cccccc}
\hline Item & Diplegia & Quadriplegia & Difference & \multicolumn{2}{c}{$\mathrm{z}$} \\
\hline 1 & $1.50 \pm .61$ & $.73 \pm .90$ & .77 & -2.317 & $.021^{*}$ \\
2 & $.33 \pm .48$ & $.09 \pm .30$ & .24 & -1.455 & .146 \\
3 & $1.00 \pm .84$ & $.36 \pm .50$ & .64 & -2.034 & $.042^{*}$ \\
4 & $.28 \pm .46$ & $.09 \pm .30$ & .19 & -1.184 & .236 \\
\hline
\end{tabular}

Values are mean \pm standard deviation ${ }^{*} \mathrm{p}<.05$

$\mathrm{V}$. As the present study focused on comparing the specifics of two gross motor skills, namely sitting balance and coor dination, we did not adjust for differences in other gross motor skills.

The total K-TIS score of the participants in the present study was 11.34 points (diplegia 15.28 points, quadriplegia 4.91 points), which amounted to $49 \%$ of the maximum (23 points). This result clearly suggests impairments to trunk control in those children with cerebral palsy. With regard to the K-TIS subscales, static sitting balance scored 4.62 points (66\%), dynamic sitting balance 4.31 (43\%), and coordination $2.41(40 \%)$; the static sitting balance score was found to be least impaired. In a study that used the TCMS and examined cerebral palsy children's postural control, Heyrman et al. [11] reported that children's static sitting balance decreased the least $(66 \%)$, consistent with the findings of the present study. The results of the TCMS and TIS suggest that it is necessary to identify problems with trunk control in children with cerebral palsy and to distinguish and compare the characteristics of impaired trunk control among different types of cerebral palsy.

The TIS subscales and total score showed a statistically significant difference between diplegic and quadriplegic children $(p<.01)$. Consistent with the results of this study, Heyrman et al. [11] reported that quadriplegic children scored significantly less than diplegic children in total TCMS score and three subscales (static sitting balance, dynamic sitting balance, and dynamic reaching) $(\mathrm{p}<.001)$. 
According to the present study's analysis of subscale scores of the two groups, static sitting balance had more items showing differences in postural control between diplegic children (6.33 points) and quadriplegic children (1.82 points). Static balance is the ability to maintain postural stability and orientation with the center of mass over the base of support while the body is at rest [18]. Static sitting balance requires predictive and compensatory postural adjustments to maintain a stable posture during movements of the upper or lower extremities while performing a task $[19,20]$. Hence, the present study's results suggest that quadriplegic children had more deficits in postural control. Upper extremity or trunk control seems to have influenced lower extremity items such as crossing legs by the therapist or the patients themselves. This means that quadriplegic children cannot use upper extremity or trunk movements in order to compensate for static sitting balance while maintaining overall balance.

The biggest differences in dynamic sitting balance between the two groups were observed in the order of Item 1 (touch seat with elbow, most affected side), Item 4 (touch seat with elbow, less affected side), Item 7 (lift pelvis from seat, most affected side), and Item 9 (lift pelvis from seat, less affected side). Items 1, 4, 7, and 9 in dynamic sitting balance are the starting positions: Item 1 is repeated as the starting position in Items 2 and 3; Item 4 in Items 5 and 6; Item 7 in Item 8; and Item 9 in Item 10. Children with spastic quadriplegia often show problems in motor planning, sensory processing, and language, which are related to learning and concept development [21]. This difference in the scores between the two groups suggests that quadriplegic children may have had an impaired ability to plan their motions and turn them into physical movement.

The trunk maintains posture, organizes the balance response, and serves as a stable base of support required to implement extremity movements [22]. The large differences in Items 1 and 4, which evaluated maintaining dynamic sitting balance during upper extremity and trunk movements, reflect the characteristics of quadriplegic children who found it difficult to control the trunk in a sitting posture and move upper and lower extremities while maintaining balance.

Diplegic children scored higher in Items 1 and 3 (rotate shoulder girdle six times and rotate pelvic girdle six times) under coordination. The coordination test, one of the TIS subscales, is designed to measure the ability to selectively rotate the upper or lower part of the trunk. Quadriplegic children who have more difficulty with trunk control would be more limited in rotating their shoulder or pelvis and returning to the original posture. The difficulties for children with cerebral palsy in performing selective movements in the sagittal plane (flexion and extention), frontal plane (lateral flexion), and transverse plan (rotation) are attributable to a lack of experience and body awareness with controlled movements in the upper and lower parts of the trunk [11]. Heyrman et al. [11] reported that quadriplegic children had limitations in performing selective movements and rotating in various directions and, more specifically, difficulties in bending the body forward. This is because bending the body forward requires support from lower extremities, and the muscles needed to be activated in the abdomen or back are lacking in quadriplegic children $[23,24]$.

The trunk control plays a crucial role in basic daily activities such as sitting, reaching, standing, walking, and eating [25]. Regardless of the severity, children with cerebral palsy have posture impairments and often prefer a sitting posture over a standing one. Nevertheless, children with cerebral palsy spend more time performing their daily tasks sitting than healthy children [26]. Sitting postural control, balance, and coordination are critical to their independent activities and overall quality of life. In general, clinical practices recognize differences in upper extremity and trunk control between diplegic and quadriplegic children and thus focus more on motion training to use hand and upper extremity skills for diplegic children than 
quadriplegic children.

To sum up the results, while both spastic diplegic children and quadriplegic children showed a decrease in sitting balance and coordination in the upper and lower parts of the trunk, diplegic children scored higher in all items under static sitting balance. Differences between the groups were observed in starting position items under dynamic sitting balance as well as items for rotation of the upper and lower parts of the trunk under coordination. Diplegic children and quadriplegic children showed clear differences in not only upper extremity functions but also static sitting balance and coordination. Differences in sitting balance and coordination between the two groups are attributable to different levels of upper trunk control and upper or lower extremity function. It is important to provide treatment to quadriplegic children by focusing on improving their static sitting balance and coordination. Diplegic children should be approached through a treatment that is more focused on dynamic sitting balance and coordination, including bending the trunk or lifting the pelvis, instead of just static sitting balance.

As a selection method and the sample size for participants in the present study were limited, it may be difficult to generalize its results to all children with cerebral palsy. In addition, as the present study did not include children with other types of spastic cerebral palsy, the results for sitting balance and overall coordination may not be applicable to all children with spastic cerebral palsy. A future study would need to examine children with various types of spastic cerebral palsy and suggest more systematic and specific results on postural control and balance. In addition, it is important to conduct a study that applies a treatment focusing on trunk control characteristics based on the type of cerebral palsy and includes the effects of the treatment. The present study holds significance as it provides a basic reference to evaluate children with cerebral palsy with regard to their postural control and balance in clinical practice, a measurement of their balance and coordination by using the K-TIS, and lastly, a determination of an applicable treatment approach.

\section{Conclusion}

The results of the present study show significant statistical differences in the overall K-TIS subscale scores and total score between diplegic children and quadriplegic children, together with differences across all items for static sitting balance between the two groups. The results suggest that diplegic children and quadriplegic children have differences in static sitting balance and coordination, along with upper extremity functions. As differences in balance and coordination originate from different levels of upper trunk control and upper or lower extremity functions, the present study finds it necessary to provide quadriplegic children with treatments focused on improving static sitting balance and coordination as well as provide diplegic children with treatments focused on dynamic sitting balance and coordination.

\section{References}

[1] Rosenbaum P, Paneth N, Leviton A, et al. A report: the definition and classification of cerebral palsy. Dev Med Child Neurol. 2007;109:8-14.

[2] Harbourne RT, Willett S, Kyvelidou A, et al. A comparison of interventions for children with cerebral palsy to improve sitting postural control: a clinical trial. Phys Ther. 2010;90(12):1881-98.

[3] Liao HF, Jeng SF, Lai JS, et al. The relation between standing balance and walking function in children with spastic diplegic cerebral palsy. Dev Med Child Neurol. 1997;39(2):106-12.

[4] Rose J, Wolff DR, Jones, et al.. Postural balance in children with cerebral palsy. Dev Med Child Neurol. 2002; 44(1):58-63.

[5] Liao HF, Mao PJ, Hwang AW. "Test-retest reliability 
of balance tests in children with cerebral palsy." Dev Med Child Neurol. 2001;43(3):180-6.

[6] Bobath B. Noteson Reflex Inhibiting Postures. Archival material. London. Bobath Centre. 1965.

[7] Brogren E, Hadders-Algra M, Forssberg H. Postural control in sitting children with cerebral palsy. Neurosci Biobehav Rev. 1998;22(4):591-6.

[8] Graaf-Peters VB, Blauw-Hospers CH, Dirks, et al. Development of postural control in typically developing children and children with cerebral palsy: possibilities for intervention? Neurosci Biobehav Rev. 2007;31(8): 1191-200.

[9] Van den Noort JC, Scholtes VA, Becher JG, et al. Evaluation of the catch in spasticity assessment in children with cerebral palsy. Arch Phys Med Rehabil. 2010;9(4): 615-23.

[10] Shumway-Cook A, Woollacott MH. Motor control Theory and practical applications (2nd ed). Philadelphia. Lippincott Williams and Wilkins, Inc. 2001.

[11] Heyrman L, Desloovere K, Molenaers G, et al. Clinical characteristics of impaired trunk control in children with spastic cerebral palsy. Res Dev Disabil. 2013;34(1): $327-34$

[12] Melhem ER, Hoon AH, Ferrucci JT, et al. Periventricular leukomalacia: relationship between lateral ventricular volume on brain MR images and severity of cognitive and motor impairment. Radiology. 2000;214(1):199-204.

[13] Sanger TD. Movement Disorders in Cerebral Palsy. J Pediatr Neurol. 2015;13(4):198-207.

[14] Verheyden G. Nieuwboer A, Mertin J, et al. The Trunk Impairment Scale: a new tool to measure motor impairment of the trunk after stroke. Clin Rehabil. 2004;18(3): 326-34.[15] Ko J, You Y. Reliability and Responsiveness of the Korean Version of the Trunk Impairment Scale for stroke patients, J Kor Phys Ther. 2015;27(4):175-82.
[16] Rannei S, Jorunn LH, Lars A, et al. Reliability and validity of the trunk impairment scale in children and adolescents with cerebral palsy. Res Dev Disabil. 2013;34(7):2075-84.

[17] Beckung E, Carlsson G, Carlsdotter S, et al. The natural history of gross motor development in children with cerebral palsy aged 1 to 15 years. Dev Med Child Neurol. 2007;49(10):751-6.

[18] Sullivan S, Leslie GP. Physical Rehabilitation (6th ed). Philadelphia. FA Davis. 2014.

[19] Bigongiari A, de Andrade e Souza F, Franciulli PM, et al. Anticipatory and compensatory postural adjustments in sitting in children with cerebral palsy. Hum Mov Sci. 2011;30(3):648-57.

[20] Girolami GL, Shiratori T, Aruin, AS. Anticipatory postural adjustments in children with hemiplegia and diplegia. J Electromyogr Kinesiol. 2011;21(6):988-97.

[21] Fernell E, Hedval, A, Norrelgen F, et al. Developmental profiles in preschool children with autism spectrum disorders referred for intervention. Res Dev Disabil. 2010;31(3):790-9.

[22] Van der Heide JC, Berger C, Fock JM, et al. Postural control during reaching in preterm children with cerebral palsy. Dev Med Child Neurol. 2004;46(4):253-66.

[23] Brogren E, Forssberg H, Hadders-Algra M. Influence of two different sitting positions on postural adjustments in children with spastic diplegia. Dev Med Child Neurol. 2001;43(8):534-46.

[24] Hadders-Algra M, Brogren E, Forssberg H. Development of postural control-differences between ventral and dorsal muscles?. Neurosci Biobehav Rev. 1998;22(4):501-6.

[25] Redstone F, West JF. The importance of postural control for feeding. Pediatr Nurs. 2004;30(2):97-100.

[26] Carlberg EB., Hadders-Algra M. Postural dysfunction in children with cerebral palsy: Some implications for therapeutic guidance. Neural Plast. 2005;12(2-3):221-8. 